\title{
The Gegenbauer Polynomial Technique: the evaluation of a class of Feynman diagrams
}

\author{
A.V.Kotikov中 \\ Laboratoire de Physique Theorique ENSLAPP \\ LAPP, B.P. 110, F-74941, Annecy-le-Vieux Cedex, France
}

\begin{abstract}
We extend Gegenbauer Polynomials technique to evaluate a class of complicated Feynman diagrams. New results in the form of ${ }_{3} F_{2}$-hypergeometrical series of unit argument, are presented. As a by-product, we present a new transformation rule for ${ }_{3} F_{2}$-hypergeometric series with argument -1 .
\end{abstract}

\footnotetext{
${ }^{1}$ On leave of absence from Particle Physics Laboratory, JINR, Dubna, Russia. e-mail: KOTIKOV@LAPPHP0.IN2P3.FR; KOTIKOV@SUNSE.JINR.DUBNA.SU
} 
Though Quantum Field Theory already has a long history and a number of different approaches, Feynman diagrams (FD) are still the main source of its dynamical information. The necessity to know more exactly about the characteristics of physical processes and specific QFT functions themselves stimulates the calculation of radiative corrections of ever higher order. This, in its turn, leads to new methods of calculation (see, for review, Ref.[1]). Fifteen years ago the method based on the expansion of propagators in Gegenbauer series (see [2]) has been introduced in [3, [4. One has shown [3] that by this method the analytical evaluation of counterterms in the minimal subtraction scheme at the 5-loop level in any model and for any composite operator was indeed possible. The Gegenbauer Polynomial (GP) technique has been applied successfully for propagator-type FD in many calculations (see [1]- [5]). In the present paper we present a new development of the GP technique and apply it to the evaluation of a class of master two-loop diagrams containing the vertex with two propagators having the powers 1 or $D / 2-1$ in D-dimensional space.

Thoughout the paper we use the following notation. The use of dimensional regularization is assumed. All the calculations are performed in the coordinate spacef of dimension $D=4-2 \varepsilon$. Any diagram is converted from $p$-space to $x$-space by applying the Fourier transform (marked by the symbol $\stackrel{f}{\Longrightarrow}$ )

$$
\frac{1}{\left(p^{2}\right)^{\nu}} \stackrel{f}{\Longrightarrow} \int \frac{d^{D} p \exp (i p x)}{\left(p^{2}\right)^{\nu}}=\pi^{D / 2} 2^{D-2 \nu} \frac{a_{0}(\nu)}{\left(x^{2}\right)^{D / 2-\nu}}, \quad a_{n}(\nu)=\frac{\Gamma(D / 2-\nu+n)}{\Gamma(\nu)},
$$

( $\Gamma$ is the Euler $\Gamma$-function) or by considering the dual diagram (see, for example, [6]). The trasformation to the dual diagram will be denoted by $\stackrel{d}{=}$.

The dual diagram is obtained from the original one by replacing $p_{i}$ by $x_{i}$ into corresponding integral and using the rules of correspondence between the graph and the integral, as in the $x$-space. Thus, after applying the Fourier transform we obtain a different $D$-space integral but the $x$-space FD similar graphically to corresponding $p$-space one. Considering the dual transformation, we do not change the integrand, except for the replacement $p_{i} \rightarrow x_{i}$, but obtain another graphical representation. Using of the both transformations leads to define the number of really independent FD (see section 2 ).

Note that contrary to [3] we analyse FD directly in $x$-space which allows us to avoid the appearence of Bessel functions. It is possible in the case of propagator-type FD, because their dependence on a single external momentum (in $p$-space) or on a single external coordinate is power-like and known beforehand. The point of interest is the coefficient function $C_{f}$, which depends on $D=4-2 \varepsilon$ and is a Laurent series in $\varepsilon$. If some diagram has $l$-lines ${ }^{3}$ with the corresponding indices $\alpha_{i}, i=\{1, \ldots, l\}$ and $L$-loops in $p$-space, then its coefficient functions in $p$-space and $x$-space are connected by

$$
C_{f}(p-\text { space })=\frac{\prod_{i=1}^{l} a_{0}\left(\alpha_{i}\right)}{a_{0}\left(\sum_{i=1}^{l} \alpha_{i}-D L / 2\right)} \cdot C_{f}(x-\text { space }) \text { and } C_{f}(p-\text { space })=C_{f}(x-\text { space })
$$

in the case of the Fourier and dual transform, respectively.

\footnotetext{
${ }^{2}$ Note that the choice of $x$-space is not crucial. If the reader prefers to work in $p$-space he can consider the variable $\left\{x_{i}, y_{i}, \ldots\right\}$ as some momenta in $p$-space.

${ }^{3}$ With the lines of the graphs there are associated power-law factors of the type $\left(x^{2}\right)^{-\alpha}$, in which $\alpha$ is called the line index.
} 
Following [3, 6] we introduce the traceless product (TP) $x^{\mu_{1} \ldots \mu_{n}}$ connected with the usual product $x^{\mu_{1}} \ldots x^{\mu_{n}}$ by the following equations (see [3, 6])

$$
\begin{aligned}
& x^{\mu_{1} \ldots \mu_{n}}=\hat{S} \sum_{p \geq 0} \frac{n !(-1)^{p} \Gamma(n-p+\lambda)}{2^{2 p} p !(n-2 p) ! \Gamma(n+\lambda)} g^{\mu_{1} \mu_{2}} \ldots g^{\mu_{2 p-1} \mu_{2 p}} x^{2 p} x^{\mu_{2 p+1}} \ldots x^{\mu_{n}} \\
& x^{\mu_{1}} \ldots x^{\mu_{n}}=\hat{S} \sum_{p \geq 0} \frac{n ! \Gamma(n-2 p+\lambda+1)}{(2)^{2 p} p !(n-2 p) ! \Gamma(n-p+\lambda+1)} g^{\mu_{1} \mu_{2}} \ldots g^{\mu_{2 p-1} \mu_{2 p}} x^{2 p} x^{\mu_{2 p+1} \ldots \mu_{n}},
\end{aligned}
$$

where hereafter $\lambda \equiv D / 2-1$.

Comparing Eqs. (A.4) and (1), we obtain the following relation between TP and GP

$$
z^{\mu_{1} \ldots \mu_{n}} x^{\mu_{1} \ldots \mu_{n}}=\frac{n ! \Gamma(\lambda)}{2^{n} \Gamma(n+\lambda)} C_{n}^{\lambda}(\hat{x} \hat{z})\left(x^{2} z^{2}\right)^{n / 2}
$$

We give also the simple but quite useful conditions:

$$
z^{\mu_{1} \ldots \mu_{n}} x^{\mu_{1} \ldots \mu_{n}}=z^{\mu_{1}} \ldots z^{\mu_{n}} x^{\mu_{1} \ldots \mu_{n}}=z^{\mu_{1} \ldots \mu_{n}} x^{\mu_{1}} \ldots x^{\mu_{n}}
$$

which follow immediately from the TP definition: $g^{\mu_{i} \mu_{j}} x^{\mu_{1} \ldots \mu_{i} \ldots \mu_{j} \ldots \mu_{n}}=0$.

The use of the TP $x^{\mu_{1} \ldots \mu_{n}}$ makes it possible to ignore terms of the type $g^{\mu_{i} \mu_{j}}$ that arise upon integration: they can be readily recovered from the general structure of the TP. Therefore, in the process of integration it is only necessary to follow the coefficient of the leading term $x^{\mu_{1}} \ldots x^{\mu_{n}}$ (see also section 3 ). The rules to integrate FD containing TP can be found, for example in [6, 7]. For a chain we have (hereafter $\left.D x \equiv\left(d^{D} x\right) /(2 \pi)^{D}\right)$ :

$$
\int D x \frac{x^{\mu_{1} \ldots \mu_{n}}}{x^{2 \alpha}(x-y)^{2 \beta}}=\frac{1}{(4 \pi)^{D / 2}} \frac{y^{\mu_{1} \ldots \mu_{n}}}{y^{2(\alpha+\beta-\lambda-1)}} A^{n, 0}(\alpha, \beta)
$$

where

$$
A^{n, m}(\alpha, \beta)=\frac{a_{n}(\alpha) a_{m}(\beta)}{a_{n+m}(\alpha+\beta-\lambda-1)}
$$

Note that in our analysis it is necessary to consider more complicate cases of integration, when the integrand contains $\Theta$ functions. Indeed, using the Eqs.(A.2) and (2), we can represent the propagator $\left(x_{1}-x_{2}\right)^{-2 \lambda}$ into the following form:

$$
\frac{1}{\left(x_{1}-x_{2}\right)^{2 \lambda}}=\sum_{n=0}^{\infty} \frac{2^{n} \Gamma(n+\lambda)}{n ! \Gamma(\lambda)} x_{1}^{\mu_{1} \ldots \mu_{n}} x_{2}^{\mu_{1} \ldots \mu_{n}}\left[\frac{1}{x_{2}^{2(\lambda+n)}} \Theta\left(x_{2}^{2}-x_{1}^{2}\right)+\left(x_{1}^{2} \longleftrightarrow x_{2}^{2}\right)\right]
$$

Using the GP properties from Appendix A and the connection (2) between GP and TP, we obtain the rules for calculating FD with the $\Theta$-terms and TP.

1. The rules have the following form:

$$
\begin{aligned}
& \int D x \frac{x^{\mu_{1} \ldots \mu_{n}}}{x^{2 \alpha}(x-y)^{2 \beta}} \Theta\left(x^{2}-y^{2}\right)=\frac{1}{(4 \pi)^{D / 2}} \frac{y^{\mu_{1} \ldots \mu_{n}}}{y^{2(\alpha+\beta-\lambda-1)}} \sum_{m=0}^{\infty} \frac{B(m, n \mid \beta, \lambda)}{m+\alpha+\beta-\lambda-1} \\
& \stackrel{(\beta=\lambda)}{=} \frac{1}{(4 \pi)^{D / 2}} \frac{y^{\mu_{1} \ldots \mu_{n}}}{y^{2(\alpha-1)}} \frac{1}{\Gamma(\lambda)} \frac{1}{(\alpha-1)(n+\lambda)}
\end{aligned}
$$




$$
\begin{aligned}
& \int D x \frac{x^{\mu_{1} \ldots \mu_{n}}}{x^{2 \alpha}(x-y)^{2 \beta}} \Theta\left(y^{2}-x^{2}\right)=\frac{1}{(4 \pi)^{D / 2}} \frac{y^{\mu_{1} \ldots \mu_{n}}}{y^{2(\alpha+\beta-\lambda-1)}} \sum_{m=0}^{\infty} \frac{B(m, n \mid \beta, \lambda)}{m+n-\alpha+\lambda+1} \\
& \stackrel{(\beta=\lambda)}{=} \frac{1}{(4 \pi)^{D / 2}} \frac{y^{\mu_{1} \ldots \mu_{n}}}{y^{2(\alpha-1)}} \frac{1}{\Gamma(\lambda)} \frac{1}{(n+\lambda+1-\alpha)(n+\lambda)}
\end{aligned}
$$

where

$$
B(m, n \mid \beta, \lambda)=\frac{\Gamma(m+\beta+n)}{m ! \Gamma(m+n+1+\lambda) \Gamma(\beta)} \frac{\Gamma(m+\beta-\lambda)}{\Gamma(\beta-\lambda)}
$$

Note that the $x$-space index of an ordinary line is $\lambda$, where all our formulae are simplified. Hereafter we add this specific case $\beta=\lambda$ to our rules.

The sum of above diagrams does not contain $\Theta$-terms and should reproduce Eq.(4). To compare the r.h.s. of Eqs.(6,7) and the r.h.s. of Eq.(包) we use the transformation of ${ }_{3} F_{2^{-}}$ hypergeometric function with unit argument (see [10]):

$$
\begin{aligned}
& { }_{3} F_{2}(a, b, c ; e, f ; 1)=\frac{\Gamma(1-a) \Gamma(e) \Gamma(f) \Gamma(c-b)}{\Gamma(e-b) \Gamma(f-b) \Gamma(1+b-a) \Gamma(c)} \cdot \\
& { }_{3} F_{2}(b, b-e+1, b-f+1 ; 1+b-c, 1+b-a ; 1)+(b \longleftrightarrow c)
\end{aligned}
$$

When $e=b+1$, the ${ }_{3} F_{2}$-function can be represented as the sum of another ${ }_{3} F_{2}$-function and a term containing only $\Gamma$-function products:

$$
\begin{aligned}
& \sum_{k=0}^{\infty} \frac{\Gamma(k+a) \Gamma(k+c)}{k ! \Gamma(k+f)} \frac{1}{k+b}=\frac{\Gamma(a) \Gamma(1-a) \Gamma(b) \Gamma(c-b)}{\Gamma(f-b) \Gamma(1+b-a)} \\
& -\frac{\Gamma(1-a) \Gamma(a)}{\Gamma(f-c) \Gamma(1+c-f)} \cdot \sum_{k=0}^{\infty} \frac{\Gamma(k+c-f+1) \Gamma(k+c)}{k ! \Gamma(k+1+c-a)} \frac{1}{k+c-b}
\end{aligned}
$$

This is the case (when $k=m, b=\alpha+\beta-\lambda-1, c=n+\beta$ ) to compare Eq.(4) and the sum of eqs.(6, (7).

Analogously to Eqs.(6) and (7) we have more complicate $\operatorname{cases}^{4}$ :

$$
\begin{aligned}
& \int D x \frac{x^{\mu_{1} \ldots \mu_{n}}}{x^{2 \alpha}(x-y)^{2 \beta}} \Theta\left(x^{2}-z^{2}\right)=\frac{1}{(4 \pi)^{D / 2}} y^{\mu_{1} \ldots \mu_{n}}\left[\frac{\Theta\left(y^{2}-z^{2}\right)}{y^{2(\alpha+\beta-\lambda-1)}} A^{n, 0}(\alpha, \beta)\right. \\
& \left.+\sum_{m=0}^{\infty} \frac{B(m, n \mid \beta, \lambda)}{z^{2(\alpha+\beta-\lambda-1)}}\left(\left(\frac{y^{2}}{z^{2}}\right)^{m} \frac{\Theta\left(z^{2}-y^{2}\right)}{m+\alpha+\beta-\lambda-1}-\left(\frac{z^{2}}{y^{2}}\right)^{m+\beta+n} \frac{\Theta\left(y^{2}-z^{2}\right)}{m-\alpha+n+1+\lambda}\right)\right] \\
& \stackrel{(\beta=\lambda)}{=} \frac{1}{(4 \pi)^{D / 2}} \frac{1}{\Gamma(\lambda)} y^{\mu_{1} \ldots \mu_{n}}\left[\frac{1}{y^{2(\alpha-1)}} \frac{\Theta\left(y^{2}-z^{2}\right)}{(\alpha-1)(n+\lambda+1-\alpha)}\right. \\
& \left.+\frac{1}{z^{2(\alpha-1)}} \frac{1}{n+\lambda}\left(\frac{\Theta\left(z^{2}-y^{2}\right)}{\alpha-1}-\left(\frac{z^{2}}{y^{2}}\right)^{n+\lambda} \frac{\Theta\left(y^{2}-z^{2}\right)}{n+1+\lambda-\alpha}\right)\right] \\
& \int D x \frac{x^{\mu_{1} \ldots \mu_{n}}}{x^{2 \alpha}(x-y)^{2 \beta}} \Theta\left(z^{2}-y^{2}\right)=\frac{1}{(4 \pi)^{D / 2}} y^{\mu_{1} \ldots \mu_{n}}\left[\frac{\Theta\left(z^{2}-y^{2}\right)}{y^{2(\alpha+\beta-\lambda-1)}} A^{n, 0}(\alpha, \beta)\right.
\end{aligned}
$$

\footnotetext{
${ }^{4}$ The full set of rules will be presented in $[8]$.
} 


$$
\begin{aligned}
& \left.-\sum_{m=0}^{\infty} \frac{B(m, n \mid \beta, \lambda)}{z^{2(\alpha+\beta-\lambda-1)}}\left(\left(\frac{y^{2}}{z^{2}}\right)^{m} \frac{\Theta\left(z^{2}-y^{2}\right)}{m+\alpha+\beta-\lambda-1}-\left(\frac{z^{2}}{y^{2}}\right)^{m+\beta+n} \frac{\Theta\left(y^{2}-z^{2}\right)}{m-\alpha+n+1+\lambda}\right)\right] \\
& \stackrel{(\beta=\lambda)}{=} \frac{1}{(4 \pi)^{D / 2}} \frac{1}{\Gamma(\lambda)} y^{\mu_{1} \ldots \mu_{n}}\left[\frac{1}{y^{2(\alpha-1)}} \frac{\Theta\left(z^{2}-y^{2}\right)}{(\alpha-1)(n+\lambda+1-\alpha)}\right. \\
& \left.-\frac{1}{z^{2(\alpha-1)}} \frac{1}{n+\lambda}\left(\frac{\Theta\left(z^{2}-y^{2}\right)}{\alpha-1}-\left(\frac{z^{2}}{y^{2}}\right)^{n+\lambda} \frac{\Theta\left(y^{2}-z^{2}\right)}{n+1+\lambda-\alpha}\right)\right]
\end{aligned}
$$

One can easily see that the sum of the above diagrams lead to results identical to (4).

2. The aim of this article is to study a class of master two-loop diagrams containing the vertex with two propagators having index 1 or $\lambda$.

Consider in the $x$-space the following general diagram

$$
\int \frac{D x D y}{y^{2 \alpha}(z-y)^{2 t}(z-x)^{2 \beta} x^{2 \gamma}(x-y)^{2 s}} \equiv J(\alpha, t, \beta, \gamma, s)
$$

and restrict ourselves to the $\mathrm{FD} A(\alpha, \beta, \gamma)=J(\alpha, \lambda, \beta, \gamma, \lambda)$, which is the one of $\mathrm{FD}$ of interest for us here. It is easily shown fo that

$$
C_{f}[A(\alpha, \beta, \gamma)]=C_{f}[A(\alpha, \sigma, \gamma)]=C_{f}[J(\gamma, \lambda, \lambda, \sigma, \alpha)]=C_{f}[J(\sigma, \gamma, \lambda, \lambda, \beta)],
$$

Doing Fourier transformation of both: the diagram $A(\alpha, \beta, \gamma)$ and its solution in the form $C_{f}[A(\alpha, \beta, \gamma)]\left(z^{2}\right)^{-\tilde{\sigma}}$, where hereafter $\tilde{t}=\lambda+1-t, t=\{\alpha, \beta, \gamma, \sigma, \ldots\}$, and considering the new diagram as one in the $x$-space (i.e. making the dual transformation) we obtain the relation

$$
\begin{aligned}
& C_{f}[A(\alpha, \beta, \gamma)] \stackrel{f}{=} \frac{a_{0}^{2}(\lambda) a_{0}(\alpha) a_{0}(\beta) a_{0}(\gamma)}{a_{0}(\delta)} C_{f}[J(\tilde{\alpha}, 1, \tilde{\beta}, \tilde{\gamma}, 1)] \\
& \stackrel{d}{=} a_{0}^{2}(\lambda) a_{0}(\alpha) a_{0}(\beta) a_{0}(\gamma) a_{0}(\sigma) C_{f}[J(\tilde{\beta}, 1, \tilde{\alpha}, \tilde{\gamma}, 1)]
\end{aligned}
$$

between the diagram, which contains the vertex with two propagators having the index $\lambda$, with the similar diagram containg the propagators with the index 1 .

Repeating the manipulations of subsection 1 we can obtain the following relations:

$$
C_{f}[J(\tilde{\beta}, 1, \tilde{\alpha}, \tilde{\gamma}, 1)]=C_{f}[J(\tilde{\beta}, 1, \tilde{\sigma}, \tilde{\gamma}, 1)]=C_{f}[J(1,1, \tilde{\gamma}, \tilde{\sigma}, \tilde{\alpha})]=C_{f}[J(\tilde{\sigma}, 1,1, \tilde{\gamma}, \tilde{\beta})]
$$

Thus, we have obtained the relations between all diagrams from the class introduced in the beginning of this section. Hence, it is necessary to find the solution for one of them. We prefer to analyse the diagram $A(\alpha, \beta, \gamma)$, that is the content of the next section.

3. We calculate the diagram $A(\alpha, \beta, \gamma)$ by the following wayĐ:

$$
A(\alpha, \beta, \gamma) \stackrel{(5)}{=} \sum_{n=0}^{\infty} \frac{2^{n} \Gamma(n+\lambda)}{n ! \Gamma(\lambda)} \int D x D y \frac{z^{\mu_{1} \ldots \mu_{n}}}{x^{2 \gamma}(z-x)^{2 \beta}} \frac{y^{\mu_{1} \ldots \mu_{n}}}{y^{2 \alpha}(x-y)^{2 \lambda}}\left[\frac{\Theta\left(z^{2}-y^{2}\right)}{z^{2(n+\lambda)}}+\frac{\Theta\left(y^{2}-z^{2}\right)}{y^{2(n+\lambda)}}\right]
$$

\footnotetext{
${ }^{5}$ For example, we can consider new FD which is the result of gluing of $A(\alpha, \beta, \gamma)$ and the propagator with index $\sigma+\Delta$, where $\sigma=3+\lambda-(\alpha+\beta+\gamma)$ and $\Delta \rightarrow 0$. This new FD has a pole $1 / \Delta$ with the corresponding coefficient independent from the external moment. Hence, this coefficient does not depend from which propagator of new FD is external and, thus, the values of these different (nongluing) FD are connected.

${ }^{6}$ The symbol $\stackrel{(n)}{=}$ markes the fact that the equation $(n)$ is used on this step.
} 
10,111 $\frac{1}{(4 \pi)^{D / 2}} \frac{1}{\Gamma(\lambda)} \frac{1}{\alpha-1} \sum_{n=0}^{\infty} \frac{2^{n} \Gamma(n+\lambda)}{n ! \Gamma(\lambda)} \int D x \frac{z^{\mu_{1} \ldots \mu_{n}} x^{\mu_{1} \ldots \mu_{n}}}{x^{2 \gamma}(z-x)^{2 \beta}} \cdot\left[\frac{1}{\lambda+n+1-\alpha}\right.$

$\left.\cdot\left(\frac{\Theta\left(z^{2}-x^{2}\right)}{z^{2(n+\lambda)} x^{2(\alpha-1)}}+\frac{\Theta\left(x^{2}-z^{2}\right)}{x^{2(n+\lambda)} z^{2(\alpha-1)}}\right)-\frac{1}{\lambda+n+\alpha-1} \cdot\left(\frac{\Theta\left(z^{2}-x^{2}\right)}{z^{2(n+\lambda+\alpha-1)}}+\frac{\Theta\left(x^{2}-z^{2}\right)}{x^{2(n+\lambda+\alpha-1)}}\right)\right]$

Evaluating the second integral and using the equation (see (A.1) and (2))

$$
x^{\mu_{1} \ldots \mu_{n}} x^{\mu_{1} \ldots \mu_{n}}=\frac{\Gamma(n+2 \lambda) \Gamma(\lambda)}{2^{n} \Gamma(2 \lambda) \Gamma(n+\lambda)},
$$

we have from Eqs.(6) and (7):

$$
\begin{aligned}
& C_{f}[A(\alpha, \beta, \gamma)]=\frac{1}{(4 \pi)^{D}} \frac{1}{\Gamma(\lambda)} \frac{1}{\alpha-1} \sum_{n=0}^{\infty} \frac{\Gamma(n+2 \lambda)}{n ! \Gamma(2 \lambda)} \sum_{m=0}^{\infty} B(m, n \mid \beta, \lambda) \\
& \cdot\left[\frac{1}{\lambda+n+1-\alpha}\left(\frac{1}{m+n+2-\alpha-\gamma+\lambda}+\frac{1}{m+n+\gamma+\beta-1}\right)\right. \\
& \left.-\frac{1}{\lambda+n+\alpha-1}\left(\frac{1}{m+n+\lambda+1-\gamma}+\frac{1}{m+n+\alpha+\gamma+\beta-2}\right)\right]
\end{aligned}
$$

The r.h.s. of Eq. (16) is already in an easy-to-use form, because the sums converge quite fastly and thus they can be easily evaluated numerically. However, the aim of our investigations is to obtain the simplest analytical expresion for the initial diagram. As we will see in this section it is ${ }_{3} F_{2}$-hypergeometric functions with unit argument.

To our purposes it is useful to transform the parts

$$
\begin{aligned}
& \left(\frac{1}{m+n+2-\alpha-\gamma+\lambda}+\frac{1}{m+n+\gamma+\beta-1}\right) \rightarrow\left(\frac{1}{m+\alpha+\gamma+\beta-\lambda-2}+\frac{1}{m+1-\gamma}\right) \\
& \left(\frac{1}{m+n+\lambda+1-\gamma}+\frac{1}{m+n+\alpha+\gamma+\beta-2}\right) \rightarrow\left(\frac{1}{m+\gamma+\beta-1-\lambda}+\frac{1}{m+2-\alpha-\gamma}\right)
\end{aligned}
$$

It is can be done by using of Eq.(9) or by representating $\Theta\left(x_{i}^{2}-x_{j}^{2}\right)$ terms into FD of the r.h.s. of Eq.(15) as $1-\Theta\left(x_{j}^{2}-x_{i}^{2}\right)$. Then the result for $C_{f}[A(\alpha, \beta, \gamma)]$ transforms to

$$
C_{f}[A(\alpha, \beta, \gamma)]=\frac{1}{(4 \pi)^{D}} \frac{1}{\Gamma(\lambda)} \frac{1}{\alpha-1}[\bar{I}-\tilde{I}]
$$

where

$$
\begin{aligned}
\bar{I} & =\sum_{n=0}^{\infty} \frac{\Gamma(n+2 \lambda)}{n ! \Gamma(2 \lambda)}\left[\frac{1}{\lambda+n+1-\alpha} \cdot\left(A^{n, 0}(\alpha-1+\gamma, \beta)+A^{n, 0}(n+\lambda+\gamma, \beta)\right)\right. \\
& \left.-\frac{1}{\lambda+n+\alpha-1} \cdot\left(A^{n, 0}(\gamma, \beta)+A^{n, 0}(n+\alpha+\lambda+\gamma-1, \beta)\right)\right]
\end{aligned}
$$

and

$$
\begin{aligned}
& \tilde{I}=\sum_{n=0}^{\infty} \frac{\Gamma(n+2 \lambda)}{n ! \Gamma(2 \lambda)} \sum_{m=0}^{\infty} B(m, n \mid \beta, \lambda)\left[\frac { 1 } { \lambda + n + 1 - \alpha } \cdot \left(\frac{1}{m+\alpha+\beta+\gamma-\lambda-2}\right.\right. \\
& \left.\left.+\frac{1}{m+1-\gamma}\right)-\frac{1}{\lambda+n+\alpha-1} \cdot\left(\frac{1}{m+\beta+\gamma-\lambda-1}+\frac{1}{m+2-\alpha-\gamma}\right)\right]
\end{aligned}
$$


Apply Eq. (9) with $k=n, b=\lambda+1-\alpha$ and with $k=m, b=\alpha+\beta+\gamma-\lambda-2$ and $b=1-\gamma$ in the r.h.s. of Eq.(18). After some algebra the double-sum terms cancel one of another and we obtain the following form for the part $\tilde{I}$

$$
\begin{aligned}
& \tilde{I}=\frac{\Gamma(1-\beta) \Gamma(\lambda+1-\alpha) \Gamma(\lambda-1+\alpha) \Gamma(1-\beta+\lambda) \Gamma(1-\gamma) \Gamma(\alpha+\beta+\gamma-\lambda-2)}{\Gamma(2 \lambda) \Gamma(2+\lambda-\alpha-\beta) \Gamma(\alpha+\gamma-1) \Gamma(2+\lambda-\gamma-\beta) \Gamma(\alpha+\beta-\lambda-1)} \\
& -\sum_{n=0}^{\infty} \frac{\Gamma(n+2 \lambda)}{n ! \Gamma(2 \lambda)} \frac{(-1)^{n} \Gamma(1-\beta)}{\Gamma(\beta-\lambda)} \cdot \frac{1}{\lambda+n+\alpha-1} \\
& \cdot\left[\frac{\Gamma(\alpha+\beta+\gamma-\lambda-2) \Gamma(2-\alpha-\gamma)}{\Gamma(3-\alpha-\beta-\gamma-n) \Gamma(\alpha+\gamma+\lambda-1+n)}+\frac{\Gamma(1-\gamma) \Gamma(\beta+\gamma-\lambda-1)}{\Gamma(\gamma-\lambda-n) \Gamma(2+2 \lambda-\beta-\gamma+n)}\right]
\end{aligned}
$$

Starting from the term $\sim(n+\lambda-1+\alpha)^{-1}$ in the r.h.s. of Eq.(18) and repeating the above analysis, we can obtain another form of $\tilde{I}$ :

$$
\begin{aligned}
& \tilde{I}=-\frac{\Gamma(1-\beta) \Gamma(\lambda+1-\alpha) \Gamma(\lambda-1+\alpha) \Gamma(1-\beta+\lambda) \Gamma(2-\alpha-\gamma) \Gamma(\beta+\gamma-\lambda-1)}{\Gamma(2 \lambda) \Gamma(\lambda+\alpha-\beta) \Gamma(\gamma) \Gamma(3+\lambda-\alpha-\gamma-\beta) \Gamma(\beta-\alpha-\lambda+1)} \\
& +\sum_{n=0}^{\infty} \frac{\Gamma(n+2 \lambda)}{n ! \Gamma(2 \lambda)} \frac{(-1)^{n} \Gamma(1-\beta)}{\Gamma(\beta-\lambda)} \cdot \frac{1}{\lambda+n-\alpha+1} \\
& \cdot\left[\frac{\Gamma(\beta+\gamma-\lambda-1) \Gamma(1-\gamma)}{\Gamma(2-\beta-\gamma-n) \Gamma(\gamma+\lambda+n)}+\frac{\Gamma(2-\alpha-\gamma) \Gamma(\alpha+\beta+\gamma-\lambda-2)}{\Gamma(\alpha+\gamma-1-\lambda-n) \Gamma(3+2 \lambda-\alpha-\beta-\gamma+n)}\right]
\end{aligned}
$$

Applying Eq.(9) to one of the two terms contained in the sum in the r.h.s. of Eqs.(19) or (20) we can have also two additional representations for $\tilde{I}$ :

$$
\begin{aligned}
\tilde{I} & =\frac{\Gamma(1-\beta) \Gamma(\lambda+1-\beta) \Gamma(\lambda+1-\alpha) \Gamma(\lambda-1+\alpha) \Gamma(1-\gamma) \Gamma(2-\alpha-\gamma)}{\Gamma(2 \lambda) \Gamma(\alpha) \Gamma(1-\alpha) \Gamma(2+\lambda-\alpha-\beta) \Gamma(3+\lambda-\alpha-\gamma-\beta)} \\
& +\sum_{n=0}^{\infty} \frac{\Gamma(n+2 \lambda)}{n ! \Gamma(2 \lambda)} \frac{(-1)^{n} \Gamma(1-\beta)}{\Gamma(\beta-\lambda)}\left[\frac{\Gamma(\alpha+\beta+\gamma-\lambda-2) \Gamma(2-\alpha-\gamma)}{\Gamma(3+2 \lambda-\alpha-\beta-\gamma+n) \Gamma(\alpha+\gamma-\lambda-1-n)}\right. \\
& \left.\cdot \frac{1}{\lambda+n-\alpha+1}-\frac{\Gamma(1-\gamma) \Gamma(\beta+\gamma-\lambda-1)}{\Gamma(\gamma-\lambda-n) \Gamma(2+2 \lambda-\beta-\gamma+n)} \cdot \frac{1}{\lambda+n+\alpha-1}\right] \\
\tilde{I}= & \frac{\Gamma(1-\beta) \Gamma(\lambda+1-\beta) \Gamma(\lambda+1-\alpha) \Gamma(\lambda-1+\alpha) \Gamma(\beta+\gamma-\lambda-1) \Gamma(\alpha+\gamma+\beta-\lambda-2)}{\Gamma(2 \lambda) \Gamma(\alpha) \Gamma(1-\alpha) \Gamma(\gamma) \Gamma(\lambda+\gamma-1)} \\
+ & \sum_{n=0}^{\infty} \frac{\Gamma(n+2 \lambda)}{n ! \Gamma(2 \lambda)} \frac{(-1)^{n} \Gamma(1-\beta)}{\Gamma(\beta-\lambda)}\left[\frac{\Gamma(\beta+\gamma-\lambda-1) \Gamma(1-\gamma)}{\Gamma(\lambda+\gamma+n) \Gamma(2-\beta-\gamma-n)} \cdot \frac{1}{\lambda+n-\alpha+1}\right. \\
- & \left.\frac{\Gamma(2-\alpha-\gamma) \Gamma(\alpha+\beta+\gamma-\lambda-2)}{\Gamma(3-\alpha-\gamma-\beta-n) \Gamma(\alpha+\lambda+\gamma-1+n)} \cdot \frac{1}{\lambda+n+\alpha-1}\right]
\end{aligned}
$$

Eqs. (19)-(22) are equal to one another because they are connected by the transformation (8). For concrete values of $\alpha, \beta$ and $\gamma$ the more convenient one of them may be used.

Thus, a quite simple solution for $A(\alpha, \beta, \gamma)$ is obtained In next section we will consider the important special case of these results.

\footnotetext{
${ }^{7}$ Before our studies, the possibility to represent $C_{f}[A(\alpha, \beta, \gamma)]$ as a combination of ${ }_{3} F_{2}$-hypergeometric functions with unit argument, has been observed in [9].
} 
4. As a simple but important example to apply these results we consider the diagram $J(1,1,1,1, \alpha)$. It arises in the framework of a number of calculations (see [11 - 15]). Its coefficient function $I(\alpha) \equiv C_{f}[J(1,1,1,1, \alpha)]$ can be found as follows

$$
I(\alpha) \stackrel{f}{=} \frac{a_{0}^{4}(1) a_{0}(\alpha)}{a_{0}(\alpha+2-2 \lambda)} C_{f}[J(\lambda, \lambda, \lambda, \lambda, \tilde{\alpha})] \text { and } C_{f}[J(\lambda, \lambda, \lambda, \lambda, \tilde{\alpha})]=C_{f}[A(\tilde{\alpha}, 3-\lambda-\tilde{\alpha}, \lambda)]
$$

The latter equation may be obtained by analogy with (14).

From Eqs. (17) and (19) we obtain

$$
\begin{aligned}
& I(\alpha)=-\frac{2}{(4 \pi)^{D}} \frac{\Gamma^{2}(\lambda) \Gamma(\lambda-\alpha) \Gamma(\alpha+1-2 \lambda)}{\Gamma(2 \lambda) \Gamma(3 \lambda-\alpha-1)} \\
& \cdot\left[\frac{\Gamma^{2}(1 / 2) \Gamma(3 \lambda-\alpha-1) \Gamma(2 \lambda-\alpha) \Gamma(\alpha+1-2 \lambda)}{\Gamma(\lambda) \Gamma(2 \lambda+1 / 2-\alpha) \Gamma(1 / 2-2 \lambda+\alpha)}+\sum_{n=0}^{\infty} \frac{\Gamma(n+2 \lambda)}{\Gamma(n+\alpha+1)} \frac{1}{n+1-\lambda+\alpha}\right]
\end{aligned}
$$

Note that in [11] Kazakov has got another result for $I(\alpha)$ :

$$
\begin{aligned}
& I(\alpha)=-\frac{2}{(4 \pi)^{D}} \frac{\Gamma^{2}(\lambda) \Gamma(1-\lambda) \Gamma(\lambda-\alpha) \Gamma(\alpha+1-2 \lambda)}{\Gamma(2 \lambda) \Gamma(\alpha) \Gamma(3 \lambda-\alpha-1)} \\
& \cdot\left[\frac{\Gamma(\lambda) \Gamma(2-\lambda) \Gamma(\alpha) \Gamma(3 \lambda-\alpha-1)}{\Gamma(2 \lambda-1) \Gamma(3-2 \lambda)}-\sum_{n=0}^{\infty}(-)^{n} \frac{\Gamma(n+2 \lambda)}{\Gamma(n+2-\lambda)}\left(\frac{1}{n+1-\lambda+\alpha}+\frac{1}{n+2 \lambda-\alpha}\right)\right]
\end{aligned}
$$

From Eqs. (23) and (24) we obtain the transformation rule for ${ }_{3} F_{2}$-hypergeometric function with argument -1 :

$$
\begin{aligned}
& { }_{3} F_{2}(2 a, b, 1 ; b+1,2-a ;-1)=b \cdot \frac{\Gamma(2-a) \Gamma(b+a-1) \Gamma(b-a) \Gamma(1+a-b)}{\Gamma(2 a) \Gamma(1+b-2 a)} \\
& -\frac{1-a}{b+a-1} \cdot{ }_{3} F_{2}(2 a, b, 1 ; b+1, b+a ; 1)-\frac{b}{1+a-b} \cdot{ }_{3} F_{2}(2 a, 1+a-b, 1 ; 2+a-b, 2-a ;-1),
\end{aligned}
$$

where $a=\lambda$ and $b=1-\lambda+\alpha$ are used.

Equation (25) has been explicitly checked at $a=1$ and $b=2-a$ (i.e. $\lambda=1$ and $\alpha=1$ ), where the ${ }_{3} F_{2}$-hypergeometric functions may be calculated exactly. We cannot directly prove Eq.(25) at arbitrary $a$ and $b$ values: the general proof seems to be non-trivial. Note that it is different from the equations of [16] and may be considered as a new transformation rule.

5. As a conclusion we note that we obtained the results for the class of master two-loop diagrams containing the vertex with two propagators, having indices 1 or $\lambda=D / 2-1$. As a by-product of the above-mentioned studies, the new transformation rule of ${ }_{3} F_{2}$-hypergeometric series with argument -1 , was obtained. It may be used together with other rules (see Ref. [16], for example).

We would like to note also, that there is only one other diagram

$$
B(\alpha, \beta, \gamma)=J(\alpha, \lambda, \beta, \lambda, \gamma)
$$

with two indices $\lambda$, which does not fall into the above-considered FD class. The diagram $J(\tilde{\alpha}, \lambda, \tilde{\beta}, \lambda, \tilde{\gamma})$ can be obtained from $B(\alpha, \beta, \gamma)$ by a analysis similar to (13) and it cannot be considered as the independent one. The incorporation of the diagram $B(\alpha, \beta, \gamma)$ in our analysis 
invites futher investigations and it is the subject of the next publication [8].

The author is grateful to Prof. A.N.Vasil'ev for a stimulation of this research and Prof. D.Broadhurst for information about a possibility to represent $\operatorname{FD} A(\alpha, \beta, \gamma)$ as a combination of ${ }_{3} F_{2}$-hypergeometric functions with unit argument (see [9]).

The author is grateful also to Prof. P.Aurenche, Prof. D.Broadhurst, Prof. K.G.Chetyrkin, Dr. A.L.Kataev, Prof. D.I. Kazakov, Dr. N.A.Kivel, Dr. A.S. Stepanenko and Prof. A.N.Vasil'ev for discussions.

\section{A Appendix}

Here we present useful formulae to use of Gegenbauer polynomials. Following [3], 1], D-space integration can be represented in the form

$$
d^{D} x=\frac{1}{2} S_{D-1}\left(x^{2}\right)^{\lambda} d x^{2} d \hat{x}
$$

where $\hat{x}=\vec{x} / \sqrt{x^{2}}$, and $S_{D-1}=2 \pi^{\lambda+1} / \Gamma(\lambda+1)$ is the surface of the unit hypersphere in $R_{D}$. The Gegenbauer polynomials $C_{n}^{\delta}(t)$ are defined as [2, 5]

$$
\left(1-2 r t+r^{2}\right)^{-\delta}=\sum_{n=0}^{\infty} C_{n}^{\delta}(t) r^{n} \quad(r \leq 1), \quad C_{n}^{\delta}(1)=\frac{\Gamma(n+2 \delta)}{n ! \Gamma(2 \delta)}
$$

whence the expansion for the propagator is:

$$
\frac{1}{\left(x_{1}-x_{2}\right)^{2 \delta}}=\sum_{n=0}^{\infty} C_{n}^{\delta}\left(\hat{x_{1}} \hat{x_{2}}\right)\left[\frac{\left(x_{1}^{2}\right)^{n / 2}}{\left(x_{2}^{2}\right)^{n / 2+\delta}} \Theta\left(x_{2}^{2}-x_{1}^{2}\right)+\left(x_{1}^{2} \longleftrightarrow x_{2}^{2}\right)\right] \text {, }
$$

where

$$
\Theta(y)= \begin{cases}1, & \text { if } y \geq 0 \\ 0, & \text { if } y<0\end{cases}
$$

Orthogonality of the Gegenbauer polynomials is expressed by the equation (see [3])

$$
\int C_{n}^{\lambda}\left(\hat{x_{1}} \hat{x_{2}}\right) \hat{x_{2}} C_{m}^{\lambda}\left(\hat{x_{2}} \hat{x_{3}}\right)=\frac{\lambda}{n+\lambda} \delta_{n}^{m} C_{n}^{\lambda}\left(\hat{x_{1}} \hat{x_{3}}\right),
$$

where $\delta_{n}^{m}$ is the Kroneker symbol.

The following formulae are useful (see [3, 6]):

$$
\begin{aligned}
C_{n}^{\delta}(x) & =\sum_{p \geq 0} \frac{(2 x)^{n-2 p}(-1)^{p} \Gamma(n-p+\delta)}{(n-2 p) ! p ! \Gamma(\delta)} \quad \text { and } \\
\frac{(2 x)^{n}}{n !} & =\sum_{p \geq 0} C_{n-2 p}^{\delta}(x) \frac{(n-2 p+\delta) \Gamma(\delta)}{p ! \Gamma(n-p+\delta+1)}
\end{aligned}
$$

Substituding the latter equation from (A.4) for $\delta=\lambda$ to the first one, we have the following equation after the separate analysis at odd and even $n$ :

$$
C_{n}^{\delta}(x)=\sum_{k=0}^{[n / 2]} C_{n-2 p}^{\lambda}(x) \frac{(n-2 k+\lambda) \Gamma(\lambda)}{k ! \Gamma(\delta)} \frac{\Gamma(n+\delta-k) \Gamma(k+\delta-\lambda)}{\Gamma(n+\lambda+1-k) \Gamma(\delta-\lambda)}
$$

This equation is an example of the equations used in our analysis. Note that alternatively to (A.5) we can use the expansion of $\left(x_{1}-x_{2}\right)^{-2 \delta}$ similar to (A.3) but directly in a series of GP $C_{n}^{\lambda}\left(\hat{x}_{1} \cdot \hat{x}_{2}\right)($ see $[4)$. 


\section{References}

[1] V.A.Smirnov, Renormalization and asymptotic expansions (Birkhaser, Basel, 1991).

[2] L.Durand, P.M.Fishbane and L.M.Simmons, J.Math.Phys. 17 (1976) 1973.

[3] K.G.Chetyrkin, A.L.Kataev and F.V.Tkachov, Nucl.Phys. B174 (1980) 447.

[4] W.Celmaster and R.J.Gonzalves, Phys.Rev. D21 (1980) 3112; A.Terrano, Phys.Lett. B93 (1980) 424.

[5] B.Lampe and G.Kramer, Phys.Scr. 28 (1986) 585.

[6] D.I.Kazakov and A.V.Kotikov, Teor.Mat.Fiz. 73 (1987) 126.

[7] D.I.Kazakov and A.V.Kotikov, Nucl.Phys. B307 (1988) 791; A.V.Kotikov, Teor.Mat.Fiz. 79 (1989) 264.

[8] A.V.Kotikov, work in progress

[9] D.Broadhurst and J.A.Gracey, preprint OUT-4102-46 (1993) (unpublished).

[10] W.N.Bailey, Generalized Hypergeometrical series (New York, 1972).

[11] D.I.Kazakov, Teor.Mat.Fiz. 62 (1985) 127.

[12] M.T.Grisaru, A.Lerda, S.Penati and D.Zanon, Nucl.Phys. B342 (1990) 564.

[13] A.N.Vasil'ev, Yu.M.Pis'mak and Yu.R.Khonkonen, Teor.Mat.Fiz. 50 (1982) 195; W.Bernrenther and F.Wegner, Phys.Rev.Lett. 57 (1986) 1382.

[14] A.N.Vasil'ev, S.E.Derkachov, N.A.Kivel and A.S.Stepanenko, Teor.Mat.Fiz. 92 (1992) 486, 94 (1993) 179; A.N.Vasil'ev and A.S.Stepanenko, Teor.Mat.Fiz. 94 (1993) 364.

[15] J.A.Gracey, IJMP A9 (1994) 567, 727.

[16] W.N.Bailey, Generalized hypergeometric series (New-York, 1972); A.P.Prudnikov, Yu.A.Brychkov and O.I.Marichev, Integrals and series Vol.3 (New-York, 1990). 